\title{
MAGI2 enhances the sensitivity of BEL-7404 human hepatocellular carcinoma cells to staurosporine- induced apoptosis by increasing PTEN stability
}

\author{
XIN LI $^{1,4}$, ZENGXIA LI $^{1-3}$, NA LI ${ }^{1}$, JINGJING QI ${ }^{1}$, KUN FAN $^{1}$, PENG YIN $^{1}$, CHAO ZHAO $^{1}$, YONGLEI LIU ${ }^{1}$, \\ WANTONG YAO $^{1}$, XIUMEI CAI ${ }^{1-3}$, LIYING WANG ${ }^{1-3}$ and XILIANG ZHA ${ }^{1-3}$ \\ ${ }^{1}$ Department of Biochemistry and Molecular Biology, School of Basic Medical Science, Fudan University; \\ ${ }^{2}$ Key Laboratory of Glycoconjugate Research, Ministry of Health; ${ }^{3}$ Key Laboratory of Molecular Medicine, \\ Ministry of Education, Shanghai 200032; ' Department of Laboratory Medicine, \\ Shanghai Public Health Clinical Center, Fudan University, Shanghai 201508 P.R. China
}

Received February 24, 2013; Accepted April 29, 2013

DOI: $10.3892 /$ ijmm.2013.1411

\begin{abstract}
Adaptor proteins are involved in the assembly of various intracellular complexes and the regulation of cellular functions. Membrane-associated guanylate kinase inverted 2 (MAGI2), also known as synaptic scaffolding molecule (S-SCAM), plays a critical role in signal transduction by assembling and anchoring its ligands. However, the role of MAGI2 in mediating apoptosis remains largely unknown. In the present study, BEL-7404 human hepatocellular carcinoma cells were transfected with a plasmid containing myc-MAGI2 or an empty plasmid and cell viability was then determined using the Cell Counting kit-8. Apoptosis was also detected using an Annexin V apoptosis assay. The cells were then treated with various doses of staurosporine (STS) for different periods of time. The overexpression of myc-MAGI2 was found to sensitize the BEL-7404 cells to apoptosis in response to STS in a time- and dose-dependent manner. Our results demonstrated that MAGI2 enhanced STS-induced apoptosis by increasing the protein expression of cytoplasmic phosphatase and tensin homologue deleted on chromosome 10 (PTEN) and decreasing its protein degradation. The apoptotic sensitivity of the cells caused by the overexpression of myc-MAGI2 was reversed by the silencing of PTEN expression by PTEN siRNA, thus revealing a momentous role of PTEN in the enhancement of the sensitivity of cancer cells to STS-induced apoptosis by MAGI2. Finally, we observed that the MAGI-PTEN complex triggered by MAGI2 overexpression reduced the phosphorylation levels
\end{abstract}

Correspondence to: Dr Liying Wang or Professor Xiliang Zha, Department of Biochemistry and Molecular Biology, Shanghai Medical College, Fudan University, 138 Yixueyuan Road, Shanghai 200032, P.R. China

E-mail: liying_wang@hotmail.com

E-mail: xlzha@shmu.edu.cn

Key words: membrane-associated guanylate kinase inverted 2, staurosporine, phosphatase and tensin homologue, AKT, apoptosis of AKT. These results suggest that MAGI2 overexpression enhances the sensitivity of cancer cells harboring ectopic PTEN to STS-induced apoptosis.

\section{Introduction}

Thousands of adaptor proteins are known in metazoans which are involved in the assembly of various intracellular complexes and the regulation of cellular functions (1). Cytoplasmic scaffold proteins may be defined as proteins that link two or more partners to enhance the efficiency of cellular signaling pathways (2). Membrane-associated guanylate kinase inverted 2 (MAGI2), also known as synaptic scaffolding molecule (S-SCAM), acts as a central organizer of multi-component signaling proteins (3). MAGI2 is highly expressed in brain tissue, which is associated with the formation and maintenance of the vertebrate central nervous system glutamatergic synapse (4). MAGI2 also exists in hepatocellular carcinoma cells as a tumor suppressor (5). MAGI2 has multiple binding partners, including the $\beta 1$-adrenergic receptor (3), $N$-methyl-D-aspartate (NMDA) glutamate receptors $(6)$, atrophin-1 $(7), \beta$ - and $\delta$-catenin $(8,9)$, membrane-associated guanylate kinase-interacting protein-1 (MAGUIN-1) (10) and phosphatase and tensin homologue deleted on chromosome 10 (PTEN) (11). MAGI2 is a multidomain adaptor protein containing a guanylate kinase (GUK) domain, two WW domains and six PSD95/Dlg/ZO1 (PDZ) domains. PDZ domain proteins play a key role in mediating key cellular functions in cells and act as adapters for interaction with the C-terminal motifs of their ligands $(12,13)$. It has been reported that PTEN interacts with MAGI2 via the PDZ-2 domains of MAGI2 and the PDZ-binding motif of PTEN $(14,15)$. PTEN is composed of an N-terminal phosphatase catalytic domain and a $\mathrm{C}$-terminal phospholipid-binding $\mathrm{C} 2$ domain. The integrity of both domains is required for full PTEN phosphatase activity (16). The expression of PTEN has been shown to suppress the growth of several human malignancies (17).

PTEN classically converts phosphatidylinositol-3,4,5trisphosphate (PIP3) in the cytoplasm to phosphatidylinositol4,5-bisphosphate (PIP2), thereby directly counteracting the 
activity of phosphatidylinositol 3 kinase (PI3K) (16). The inactivation of PTEN results in the constitutive activation of the PI3K/AKT pathway and in cell cycle progression, migration and survival $(18,19)$. In our previous study, we showed that PTEN plays a critical role in the MAGI2-induced inhibition of cell migration and proliferation in human hepatocellular carcinoma cells (5).

The PTEN-MAGI2 complex promotes a series of biological functions; however, the role of the complex in mediating apoptosis is unknown. In present study, we found that cytoplasmic PTEN is involved in the enhancement of the sensitivity of BEL-7404 human hepatocellular carcinoma cells to staurosporine (STS)-induced apoptosis by MAGI2. STS, a potent inhibitor of protein kinase $\mathrm{C}$, is used in vitro as an initiator of apoptosis in a wide variety of cell types (20-22). The mechanism by which STS induces apoptosis has not yet been fully elucidated. In present study, we demonstrate that MAGI2 enhances STS-induced apoptosis by increasing PTEN stability and downregulating the phosphorylation level of AKT in a hepatocellular carcinoma cell line.

\section{Materials and methods}

Cell culture and cycloheximide (CHX) treatment. BEL-7404 human hepatocellular carcinoma cells were maintained in Dulbecco's modified Eagle's medium (DMEM; Gibco, Carlsbad, CA, USA) supplemented with $10 \%$ fetal bovine serum (Gibco) in a $37^{\circ} \mathrm{C}$ incubator with $5 \% \mathrm{CO}_{2}$. The cells were treated with $\mathrm{CHX}(100 \mu \mathrm{g} / \mathrm{ml})$ for different periods of time and incubated for $10 \mathrm{~h}$ with different doses of $\mathrm{CHX}$. Cellular proteins were extracted, cell lysates were obtained, total protein concentrations were determined using the Lowry method and western blot analysis was preformed.

Antibodies and reagents. Anti-PARP monoclonal antibody and LY294002 were purchased from Cell Signaling Technology, Inc. (Danvers, MA, USA). Anti-PTEN antibody was purchased from BD Biosciences (Bedford, MA, USA). Anti-myc, antiMAGI2 and the translation inhibitor, $\mathrm{CHX}$, were obtained from Sigma-Aldrich (St. Louis, MO, USA). STS was obtained from Roche Applied Science (Indianapolis, IN, USA). Anti- $\beta$-actin, anti- $\alpha$-tubulin monoclonal antibody, peroxidase-conjugated affinity goat anti-rabbit IgG and goat anti-mouse IgG secondary antibodies were purchased from Kangchen Biotech Co., Ltd. (Shanghai, China). The enhanced chemiluminescence (ECL) assay kit was commercially available from Tiangen Biotech Co., Ltd. (Shanghai, China). Alexa Fluor 488-conjugated goat anti-mouse IgG and $\mathrm{Cy} 3$-conjugated goat anti-rabbit IgG secondary antibodies were purchased from Beyotime (Jiangsu, China). DAPI (4',6-diamidino-2-phenylindole) was purchased from Vector Laboratories. Lipofectamine ${ }^{\mathrm{TM}} 2000$ was obtained from Invitrogen (Carlsbad, CA, USA). Opti-MEM medium was purchased from Gibco. FBS was purchased from PAA Laboratories GmbH (Haidmannweg, Austria). The Annexin V-FITC Apoptosis Detection kit and the Cell Counting kit-8 (CCK-8) were purchased from Beyotime.

Transient cell transfection. pCl-neo-myc-MAGI2 and pCl-neo empty vector plasmids were generous gifts from Professor Yoshimi Takai (6). Cells were plated on 6-well plates. When cells grew to $90-95 \%$ confluence, the two plasmids were transfected into the cells using Lipofectamine 2000 according to the manufacturer's instructions. After $7 \mathrm{~h}$ of transfection, the medium was changed with fresh medium containing $10 \% \mathrm{FBS}$ and the cells were incubated at $37^{\circ} \mathrm{C}$ for the appropriate amount of time.

Cell viability assay. The cells were plated at a density of $2 \times 10^{5}$ cells/well in a 96-well plate and cultured for $12 \mathrm{~h}$. Following treatment with DMSO or $1 \mu \mathrm{M}$ STS for different periods of time, cell viability was determined using the CCK-8. Subsequently, $5 \mu$ of CCK- 8 solution mixed with $100 \mu$ l culture medium were added to each well, and the cells were incubated for $0.5-1 \mathrm{~h}$ at $37^{\circ} \mathrm{C}$. The absorbance was then measured at $450 \mathrm{~nm}$ using an ELISA reader.

Annexin V apoptosis assay. The Annexin V-FITC Apoptosis Detection kit was used to detect apoptosis. According to the manufacturer's instructions, the cells were washed twice with cold phosphate-buffered saline (PBS) and resuspended at a concentration of $1 \times 10^{5}$ cells $/ \mathrm{ml}$. After the addition of $195 \mu \mathrm{l}$ of binding buffer, $5 \mu \mathrm{l}$ Annexin V-FITC were added and the cells were incubated for $10 \mathrm{~min}$ at room temperature. After the addition of $190 \mu \mathrm{l}$ of binding buffer, $10 \mu \mathrm{l}$ propidium iodide (PI) were added and the cells were placed on ice in the dark. The samples were then analyzed by flow cytometry within $1 \mathrm{~h}$.

RNA isolation and reverse transcription-polymerase chain reaction $(R T-P C R)$. Total RNA was isolated using TRIzol reagent following the manufacturer's instructions. Oligo(dT)18 primer and M-MLV reverse transcriptase were used for the first strand synthesis. For semi-quantitative RT-PCR, the first primer pair to PTEN was 5'-CAGAAAGACTTGAAGGCGTAT-3' and 5'-AACGGCTGAGGGAACTC-3'. The second primer pair to $\beta$-actin was 5'-TGATGATATCGCCGCGCTCGTCGT-3' and 5'-CACAGCCTGGATAGCAACGTACAT-3'. $\beta$-actin was used as the internal control.

siRNA transfection. The knockdown of PTEN was performed using siRNA (synthesized by GenePharma, Shanghai, China). The target sequences were displayed as follows: PTEN sense, 5'-AGGCACAAGAGGCCCUAGAUU-3' and antisense, 5'-UCUAGGGCCUCUUGUGCCUUU-3'. Transfection was performed using Lipofectamine 2000 according to the manufacturer's instructions.

Western blot analysis. The cells were lysed in $1 \mathrm{X}$ sodium dodecyl sulfate (SDS) lysis buffer (50 mM Tris-HCl, pH 6.8, $2 \%$ SDS, $10 \%$ glycerol, $100 \mathrm{mM} \mathrm{Na}_{3} \mathrm{VO}_{4}$ and $1 \mathrm{mM}$ PMSF). Protein concentration was determined using the Lowry method. Equal amounts of protein were separated by SDS-PAGE and electrophoretically transferred onto PVDF membranes. The membranes were blocked with $5 \%$ fat-free milk with $0.05 \%$ Tween-20 in PBS. After probing with the primary and horseradish peroxidase (HRP)-conjugated secondary antibodies, the membranes were visualized by ECL.

Immunofluorescence and confocal microscopy. The cells were washed with cold PBS (pH 7.4) and fixed with 4\% paraformaldehyde for $20 \mathrm{~min}$, then permeabilized with $0.1 \%$ Triton X-100 
for 6 min on ice. After blocking with 3\% bovine serum albumin (BSA) for $1 \mathrm{~h}$ at room temperature, the cells were incubated with anti-MAGI2 antibody and anti-PTEN antibody for $3 \mathrm{~h}$ at $37^{\circ} \mathrm{C}$, and then incubated with $\mathrm{Cy} 3$-conjugated goat antirabbit IgG secondary antibody and Alexa Fluor 488-conjugated goat anti-mouse IgG secondary antibody for $2 \mathrm{~h}$ at $37^{\circ} \mathrm{C}$ in the dark. The nuclei were stained with DAPI for $10 \mathrm{~min}$ at room temperature in the dark. The immunofluorescence-stained cells were visualized under a laser confocal microscopy (Leica SP5; Leica Microsystems, Wetzlar, Germany) and the resulting data were analyzed using Leica AF6000 software.

\section{Results}

BEL-7404 hepatocellular carcinoma cells transfected with myc-MAGI-2 have lower cell viability. It has been reported that MAGI2 is expressed mainly in neuronal cells. As shown in our previous study, MAGI2 also exists in hepatocarcinama cells as a tumor suppressor (5). In this study, we detected the expression of MAGI2 in four hepatocellular carcinoma cell lines and two human normal liver cells. The results are shown in Fig. 1A. Human normal liver cells and hepatocellular carcinoma cells expressed MAGI2 protein, but the band was slight apart from the SMMC-7721 cells. We selected the BEL-7404 cells for further study since their endogenous native MAGI2 expression was lower. The BEL-7404 cells were transfected with the empty vector or the myc-MAGI2 expression plasmid, and were designated as vector-7404 and myc-MAGI2-7404 cells, respectively (Fig. 1B). The results revealed that the mycMAGI2 plasmid was successfully transfected into the cells, and endogenous native MAGI2 expression was not observed. To determine whether MAGI2 regulates STS-induced apoptosis, cell viability was determined using the CCK-8. The cell viability of the myc-MAGI2-7404 cells was lower than that of the vector-7404 cells following stimulation with STS (Fig. 1C). These results demonstrate that MAGI 2 enhances the sensitivity of the cells to STS in a time-dependent manner, thus aiding in the decrease in cell viability.

MAGI2 enhances STS-induced apoptosis in a time- and dosedependent manner. We performed a series of experiments to explore the potential role of MAGI2 in cell apoptosis induced by STS (Fig. 2). First, nuclear fragmentation was more easily observed in the myc-MAGI2-7404 cells than in the vector-7404 cells as the treatment ( $1 \mu \mathrm{M}$ STS) time increased, as shown by DAPI staining. Nuclear staining by DAPI showed a much stronger apoptotic response in the myc-MAGI2-7404 cells compared to the vector-7404 cells (Fig. 2A). As shown in Fig. 2B, Annexin V/PI staining indicated that the percentage of apoptotic cells was much higher in the myc-MAGI2-7404 cells than in the vector-740 4 cells after $4 \mathrm{~h}$. The proteolytic cleavage of poly(ADP-ribose) polymerase (PARP) was also examined by western blot analysis. Treatment of the cells with $1 \mu \mathrm{M} \mathrm{STS}$ caused the time-dependent proteolytic cleavage of PARP, with the diminution and even the disappearance of full-length PARP and the appearance of large fragments. A significant cleavage of PARP was observed in the myc-MAGI2-7404 cells, which enhanced STS-induced apoptosis (Fig. 2C). These data demonstrate that myc-MAGI2 overexpression enhances STS-induced apoptosis in a time-dependent manner. To further assess the role
$\mathbf{A}$

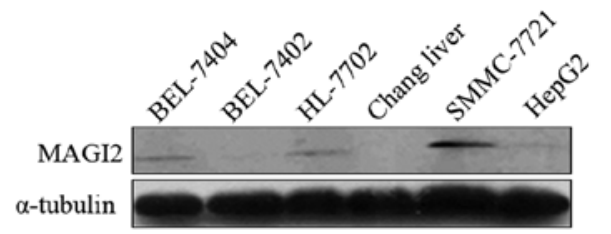

$\mathbf{B}$
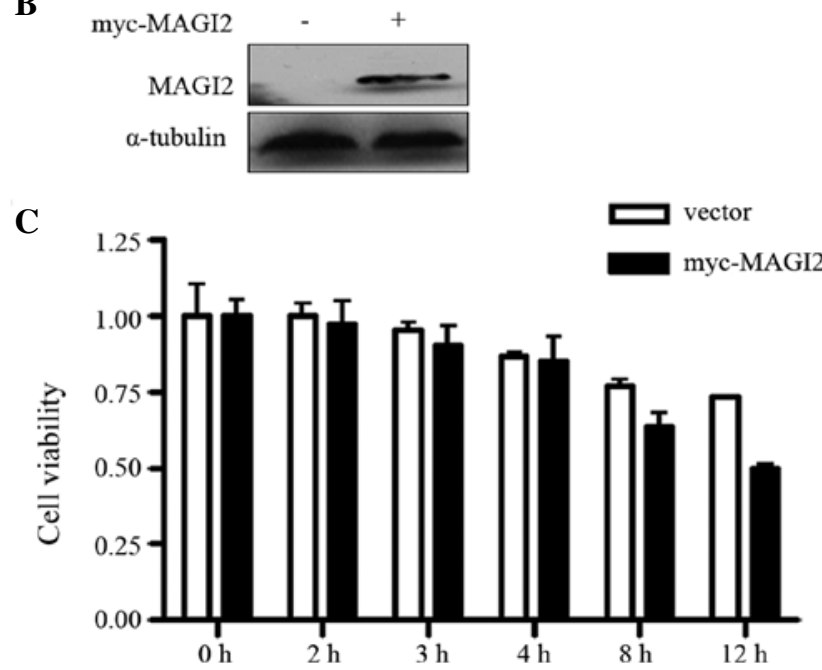

Figure 1. Membrane-associated guanylate kinase inverted 2 (MAGI2) is expressed at lower levels in hepatocellular carcinoma cell lines, and transfection with myc-MAGI2 inhibits cell proliferation. (A) The expression of MAGI2 in four hepatocellular carcinoma cell lines and two human normal liver cells. (B) Western blot analysis with anti-MAGI 2 and anti- $\alpha$-tubulin antibodies following transfection with myc-MAGI2 in BEL-7404 cells. (C) Cell viability was examined in the vector-7404 and myc-MAGI2-7404 cells following treatment with $1 \mu \mathrm{M}$ staurosporine (STS) for different periods of time. The experiment was carried out at least three times.

of MAGI2 in STS-induced apoptosis, we examined apoptosis in the vector-7404 and myc-MAGI2-7404 cells by Annexin V/PI staining after treatment of the cells with various doses of STS for $6 \mathrm{~h}$ (Fig. 2D). It was found that compared with the vector cells, treatment with STS significantly increased cell apoptosis in a dose-dependent manner in the myc-MAGI 2 cells. The cells were then treated with various doses of STS for $4 \mathrm{~h}$. At each concentration of STS, the vector-7404 cells had lower amounts of cleaved PARP compared with the myc-MAGI2-7404 cells (Fig. 2E). These results illustrate that MAGI2 enhances apoptosis induced by STS in a time- and dose-dependent manner.

MAGI2 enhances STS-induced apoptosis by upregulating PTEN protein expression and decreasing its degradation. We examined the relative expression level of PTEN in the vector-7404 and myc-MAGI2-7404 cells treated with STS for different periods of time by western blot analysis. The result revealed a dramatic elevation of PTEN levels in the myc-MAGI2-7404 cells in a time-dependent manner (Fig. 3A). We then examined the PTEN protein level in the myc-MAGI2-7404 and vector7404 cells treated with various concentrations of STS. As shown in Fig. 3B, the expression of PTEN in the myc-MAGI2-7404 and vector-7404 cells was similar. The mRNA level of PTEN was then detected; no significant change in the PTEN mRNA level was observed in the vector-7404 and myc-MAGI2-7404 cells treated with various doses of STS for different amounts 
A

STS $(1 \mu \mathrm{M})$
vector

$0 \mathrm{~h}$

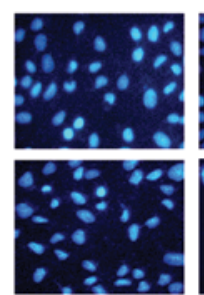

$2 \mathrm{~h}$

$3 \mathrm{~h}$
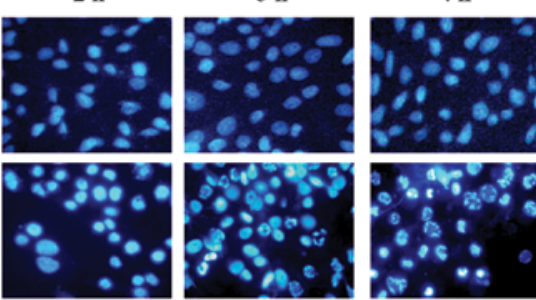

B

myc-MAGI2
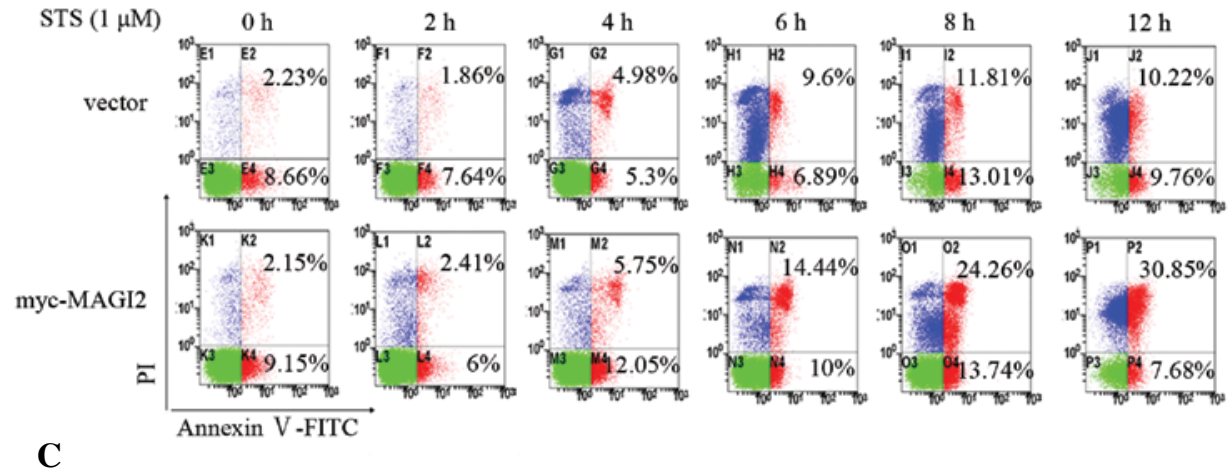

$\begin{array}{rccccccccccccc}\text { myc-MAGI2 } & - & - & - & - & - & - & + & + & + & + & + & + \\ \text { STS }(1 \mu \mathrm{M}) & 0 \mathrm{~h} & 2 \mathrm{~h} & 4 \mathrm{~h} & 6 \mathrm{~h} & 8 \mathrm{~h} & 12 \mathrm{~h} & 0 \mathrm{~h} & 2 \mathrm{~h} & 4 \mathrm{~h} & 6 \mathrm{~h} & 8 \mathrm{~h} & 12 \mathrm{~h}\end{array}$

D
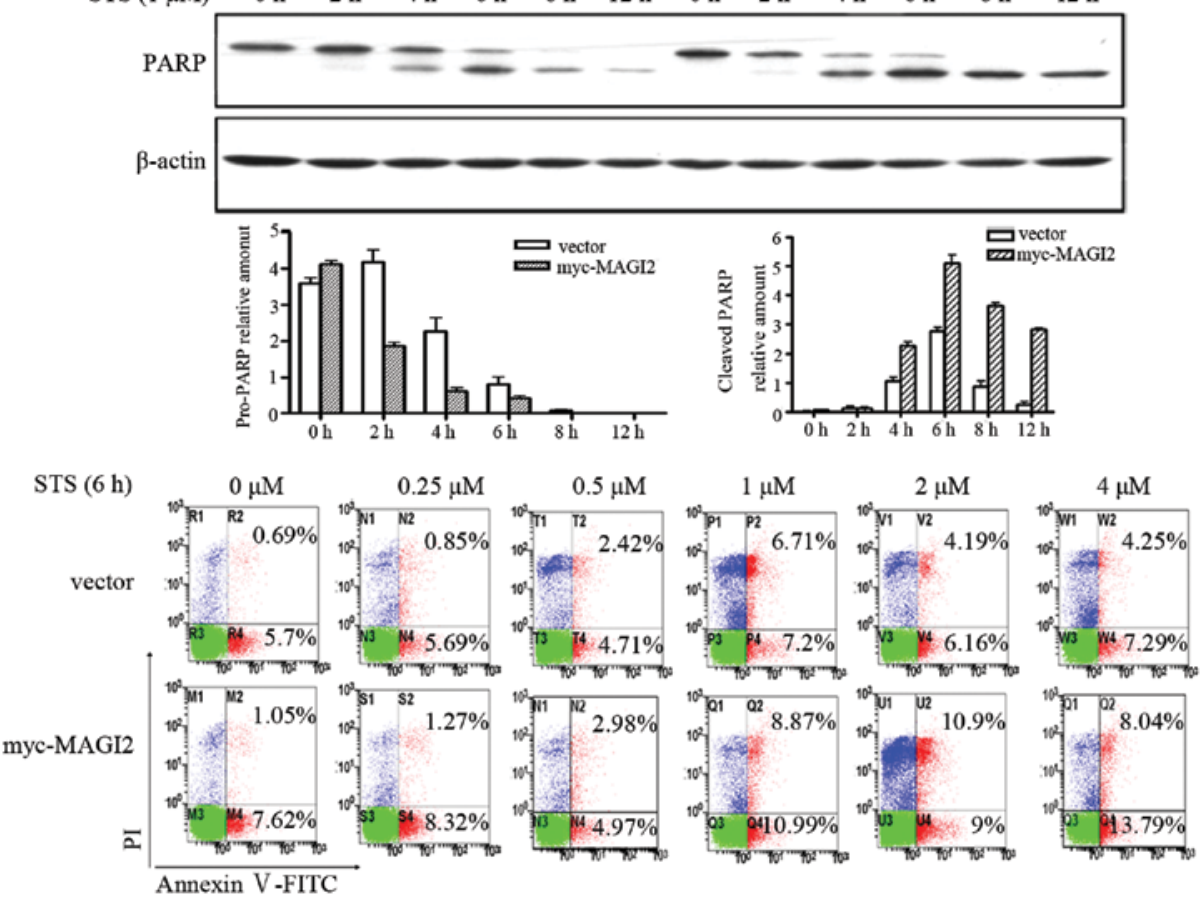

$\mathbf{E}$
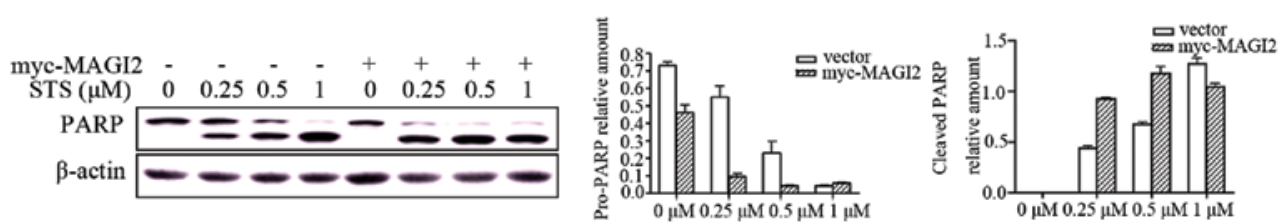

Figure 2. Membrane-associated guanylate kinase inverted 2 (MAGI2) promotes apoptosis following treatment with staurosporine (STS). (A) After the cells were treated with $1 \mu \mathrm{M} \mathrm{STS}$ for different periods of time, the nuclear condensation and fragmentation of apoptotic cells were increased in the myc-MAGI2-7404 cells. This result shown is from three independent experiments. (B) Evaluation of apoptosis of the vector-7404 and myc-MAGI2-7404 cells treated with $1 \mu \mathrm{M}$ STS for different periods of time using Annexin V/PI staining. Cells were double stained with Annexin-V-FITC and propidium iodide (PI) and apoptosis was analyzed by flow cytometry. Annexin- $\mathrm{V}^{-}, \mathrm{PI}^{-}$cells were live cells, Annexin- $\mathrm{V}^{+}, \mathrm{PI}^{-}$cells were early apoptotic cells and Annexin- $\mathrm{V}^{+}, \mathrm{PI}^{+}$cells were late apoptotic cells. This result shown is from three independent experiments. (C) Total cell extracts were collected from the vector-7404 and myc-MAGI2-7404 cells at different time points (0, $2,4,6,8$ and $12 \mathrm{~h}$ ) after treatment with $1 \mu \mathrm{M}$ STS, and were then examined using anti-PARP antibodies. $\beta$-actin was used as the loading control. The bottom chart is the analysis of the quantitative ratios of pro-PARP and cleaved-PARP, relative to the amount of $\beta$-actin for the data mentioned above. Each bar represents the mean \pm SD of three independent experiments. (D) Evaluation of apoptosis of the vector-7404 and myc-MAGI2-7404 cells treated with different concentrations of STS using Annexin V/PI staining. Cells were treated with various doses of STS for $6 \mathrm{~h}$. Cells were double stained with Annexin-V-FITC and PI and apoptosis was analyzed by flow cytometry. (E) Total cell extracts were collected from the vector-7404 and myc-MAGI2-7404 cells treated with various doses of STS after $4 \mathrm{~h}$, and were then examined using anti-PARP antibodies. $\beta$-actin was used as the loading control. The chart shows the analysis of the quantitative ratios of pro-PARP and cleaved PARP, relative to the amount of $\beta$-actin for the data mentioned above. Each bar represents the mean \pm SD of three independent experiments. 
A
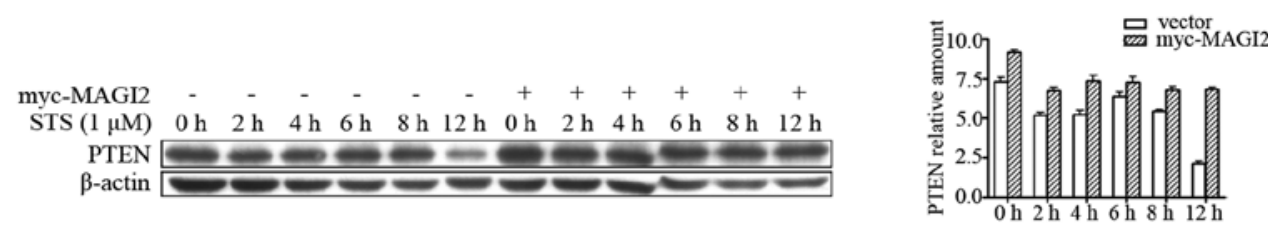

B
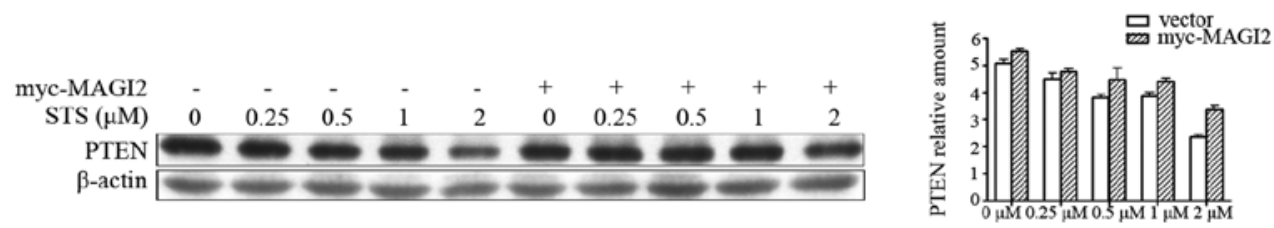

C

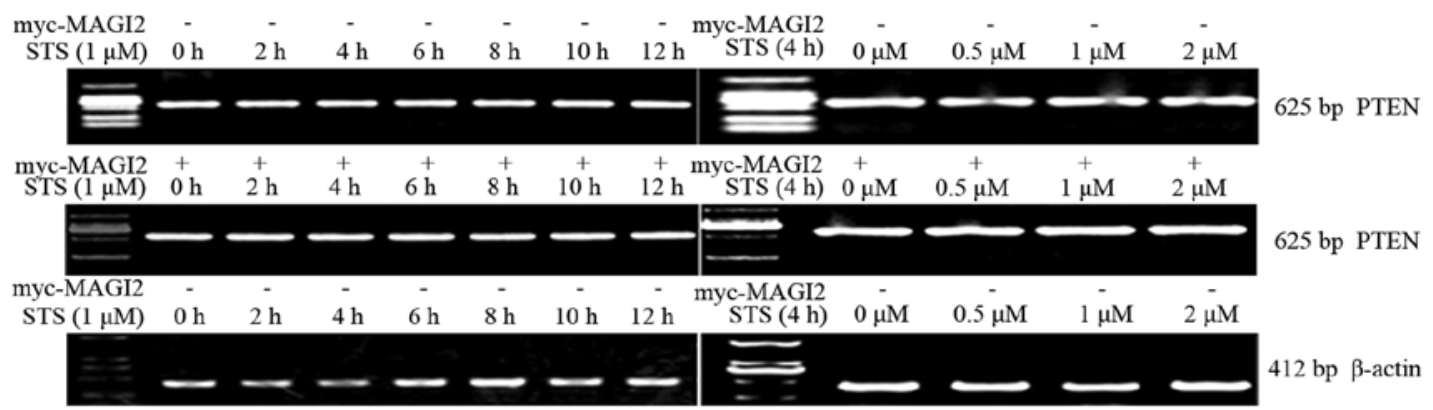

D
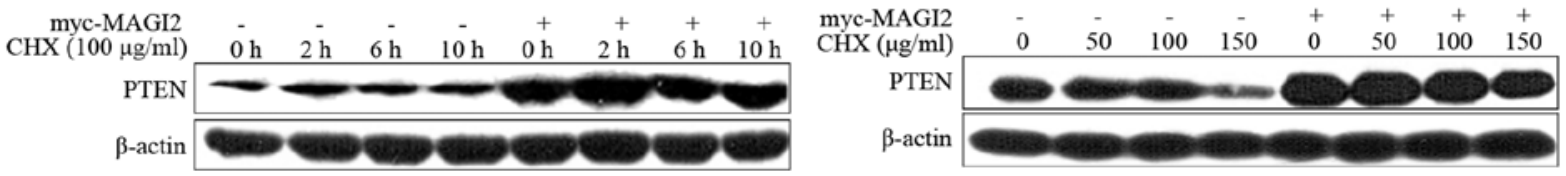

Figure 3. Membrane-associated guanylate kinase inverted 2 (MAGI2) increases phosphatase and tensin homologue (PTEN) protein levels following treatment with staurosporine (STS). (A) The vector-7404 and myc-MAGI2-7404 cells were incubated with STS for different periods of time, and the protein level of PTEN was measured by western blot analysis using anti-PTEN antibody. The chart on the right is the analysis of the quantitative ratio of PTEN relative to the amount of $\beta$-actin for the data mentioned above. Each bar represents the mean \pm SD of three independent experiments. (B) The vector-7404 and myc-MAGI2-7404 cells were incubated with various doses of STS and the protein level of PTEN was measured by western blot analysis. The chart on the right is the analysis of the quantitative ratio of PTEN relative to the amount of $\beta$-actin for the data mentioned above. Each bar represents the mean \pm SD of three independent experiments. (C) PTEN mRNA level was determined by RT-PCR in the vector-7404 and myc-MAGI2-7404 cells treated with various doses of STS for different periods of time. (D) Cells were exposed to $100 \mu \mathrm{g} / \mathrm{ml}$ cycloheximide (CHX) for different periods of time (0, 2, 6 and $10 \mathrm{~h})$. Quantitative representation of PTEN protein normalized to $\beta$-actin. (E) Cells were treated with different doses $(0,50,100$ and $150 \mu \mathrm{g} / \mathrm{ml})$ of CHX for $15 \mathrm{~h}$. Quantitative representation of PTEN protein normalized to $\beta$-actin.

of time (Fig. 3C). To further explore the mechanism of PTEN upregulation, the vector-7404 and myc-MAGI2-7404 cells were incubated with CHX (an inhibitor of protein synthesis), and then PTEN protein levels were detected at different time points (Fig. 3D) and different dose points (Fig. 3E) in the presence of $\mathrm{CHX}$. The degradation rate of PTEN was slower in the mycMAGI2-7404 cells (Fig. 3D and E) than in the control cells. Altered PTEN levels triggered by MAGI2 correlated with the rate of apoptosis, suggesting that MAGI2 enhanced STS-induced apoptosis by decreasing PTEN protein degradation. These data indicate that MAGI2 augments the protein stability of PTEN in BEL-7404 cells. Therefore, MAGI2 enhances STS-induced apoptosis by increasing the PTEN protein level and decreasing its degradation.

Downregulation of PTEN inhibits STS-induced apoptosis. In order to further confirm that PTEN participates in the enhancement of STS-induced apoptosis by MAGI2, we performed a series of experiments after the knockdown of PTEN by PTEN siRNA.
The results of DAPI staining indicated that the STS-induced nuclear fragmentation in the myc-MAGI2-7404 cells was noticeably reduced when the PTEN gene was knocked down (Fig. 4A). Annexin V/PI staining verified that myc-MAGI2 alone had a significant effect on the induction of apoptosis, whereas transfection with the myc-MAGI2 plasmid in conjunction with PTEN siRNA rescued the cells from STS-induced apoptosis (Fig. 4B). Western blot analysis was then performed to detect the PTEN protein level and the cleavage of PARP. Transfection with PTEN siRNA markedly suppressed the protein level of PTEN, and successfully inhibited the STS-induced PARP cleavage in the myc-MAGI2-7404 cells (Fig. 4C, lanes 6 and 8). These results suggested that the MAGI2-enhanced apoptosis was blocked by the knockdown of the PTEN gene. Thus, PTEN was a critical signaling molecule involved in the apoptosis induced by STS in myc-MAGI2-7404 cells.

Co-localization of MAGI2 and PTEN in response to MAGI2 overexpression. To better define the link between MAGI 2 and 


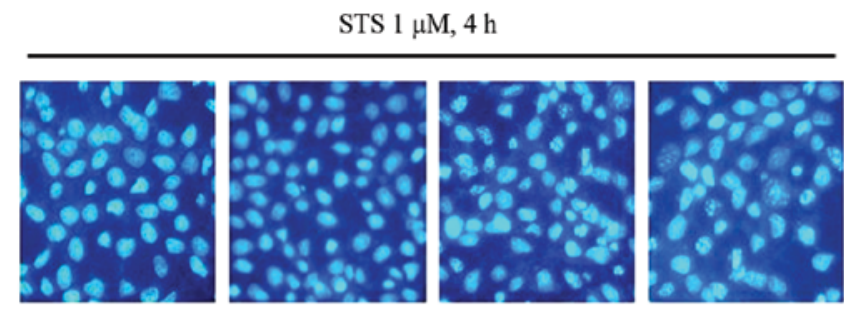

vector+NC vector+PTEN SiRNA MAGI2+NC MAGI2+PTEN SiRNA

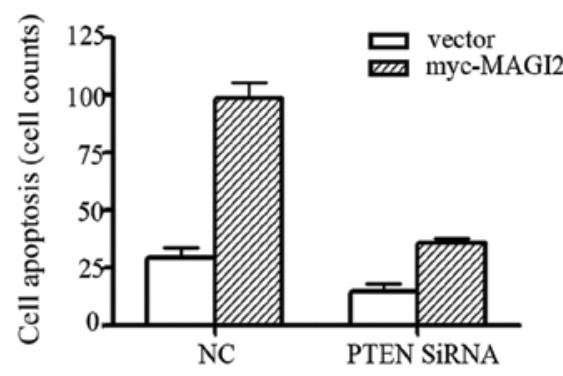

C

B

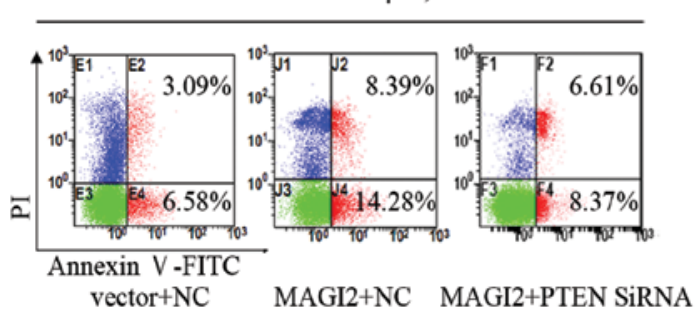

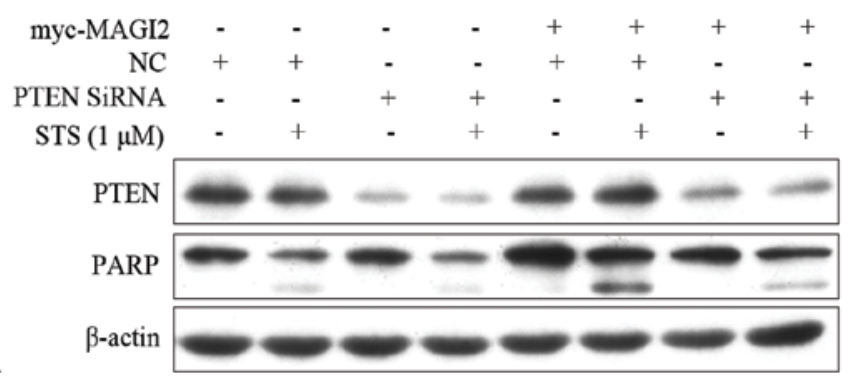

Figure 4. The staurosporine (STS)-induced apoptosis enhanced by membrane-associated guanylate kinase inverted 2 (MAGI2) was blocked by the knockdown of phosphatase and tensin homologue (PTEN). (A) Downregulation of PTEN inhibited STS-induced DNA fragmentation in myc-MAGI2-7404 cells. Cells were transfected with vector + negative control (NC), vector + PTEN siRNA, MAGI2 + NC, or MAGI2 + PTEN siRNA and treated with $1 \mu \mathrm{M}$ STS for $4 \mathrm{~h}$ The chart on the right is the analysis of the number of apoptotic cells. The result shown is from three independent experiments. (B) Downregulation of PTEN inhibited STS-induced cell apoptosis in myc-MAGI2-7404 cells. Cells were transfected with vector + NC, MAGI2 + NC, or MAGI2 + PTEN siRNA and treated with $1 \mu \mathrm{M}$ STS for $4 \mathrm{~h}$, and then assessed by Annexin V/PI staining. The result shown is from three independent experiments. (C) Vector-7404 cells and myc-MAGI2-7404 cells were transfected with NC or PTEN siRNA, and incubated with $1 \mu \mathrm{M}$ STS for $4 \mathrm{~h}$, then the protein levels of PTEN and PARP were measured by western blot analysis.
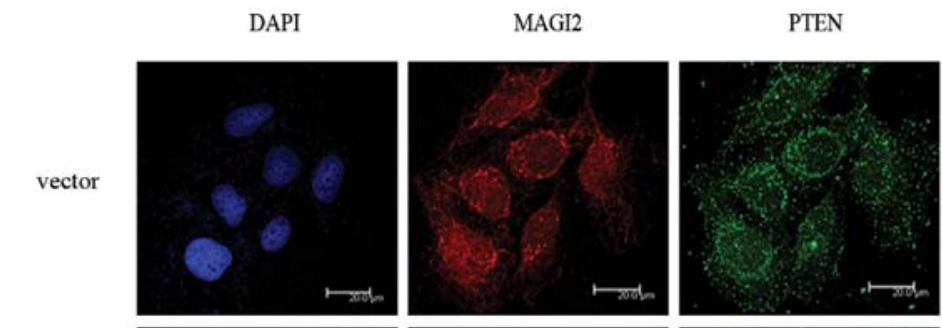

MAGI2 \& PTEN
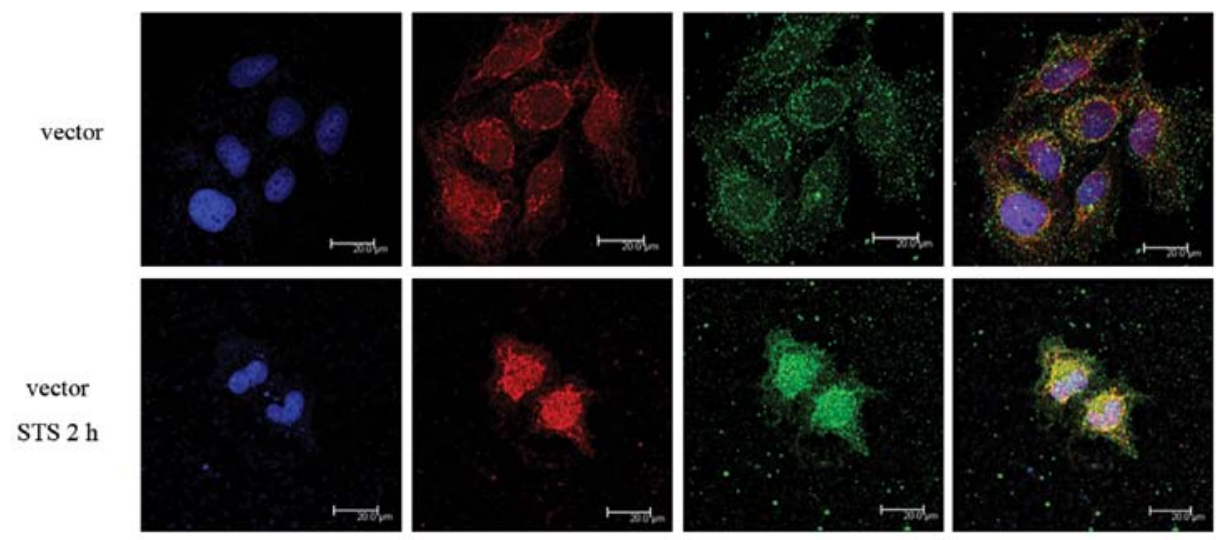

MAGI2
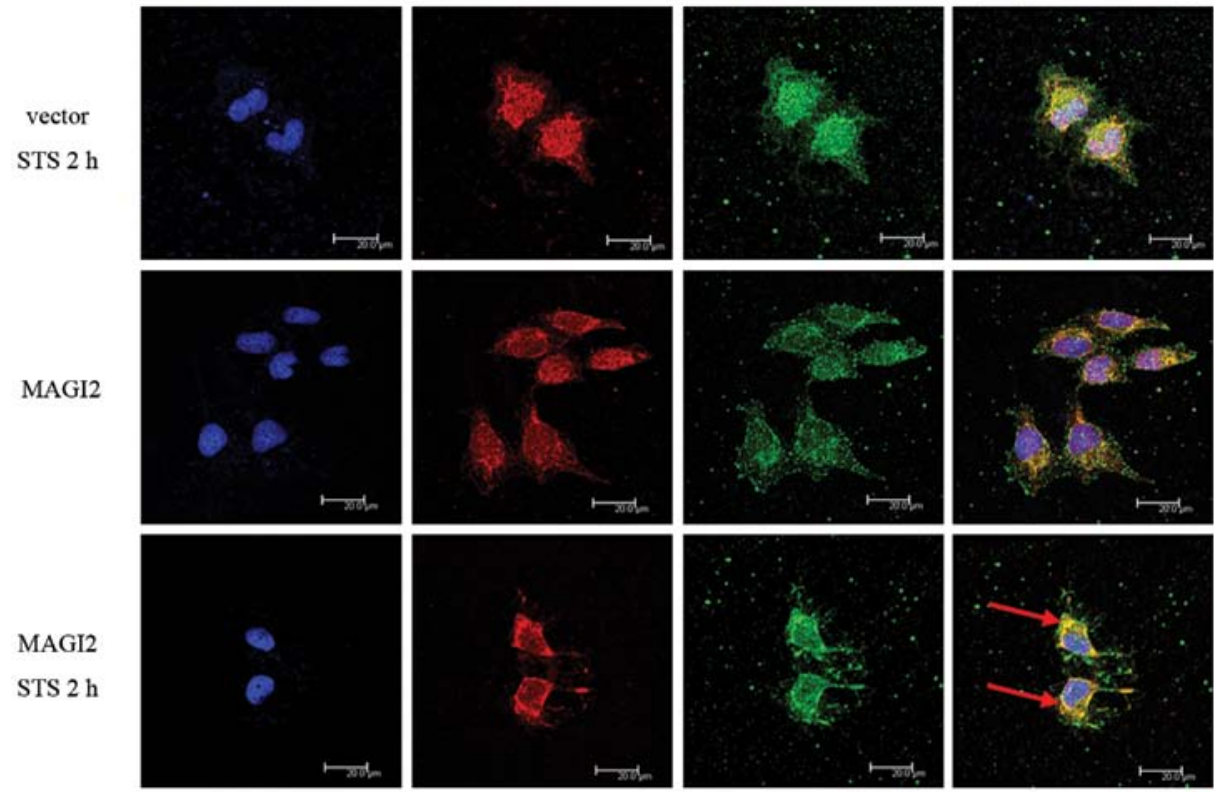

Figure 5. The co-localization of membrane-associated guanylate kinase inverted 2 (MAGI2) and phosphatase and tensin homologue (PTEN) was strengthened following treatment with staurosporine (STS). Vector-7404 and myc-MAGI2-7404 cells were treated with $1 \mu \mathrm{M} \mathrm{STS}$ for 0-2 h and then stained with antiMAGI2 and anti-PTEN antibodies and DAPI, and then assayed under a confocal immunofluorescence microscope. The subcellular localization of MAGI2 (red), PTEN (green) and nuclei (blue) is shown. Cells were visualized under a laser confocal microscope at $63 * \mathrm{Z} 2$ magnification and the resulting data were analyzed using Leica AF6000 software. 
A
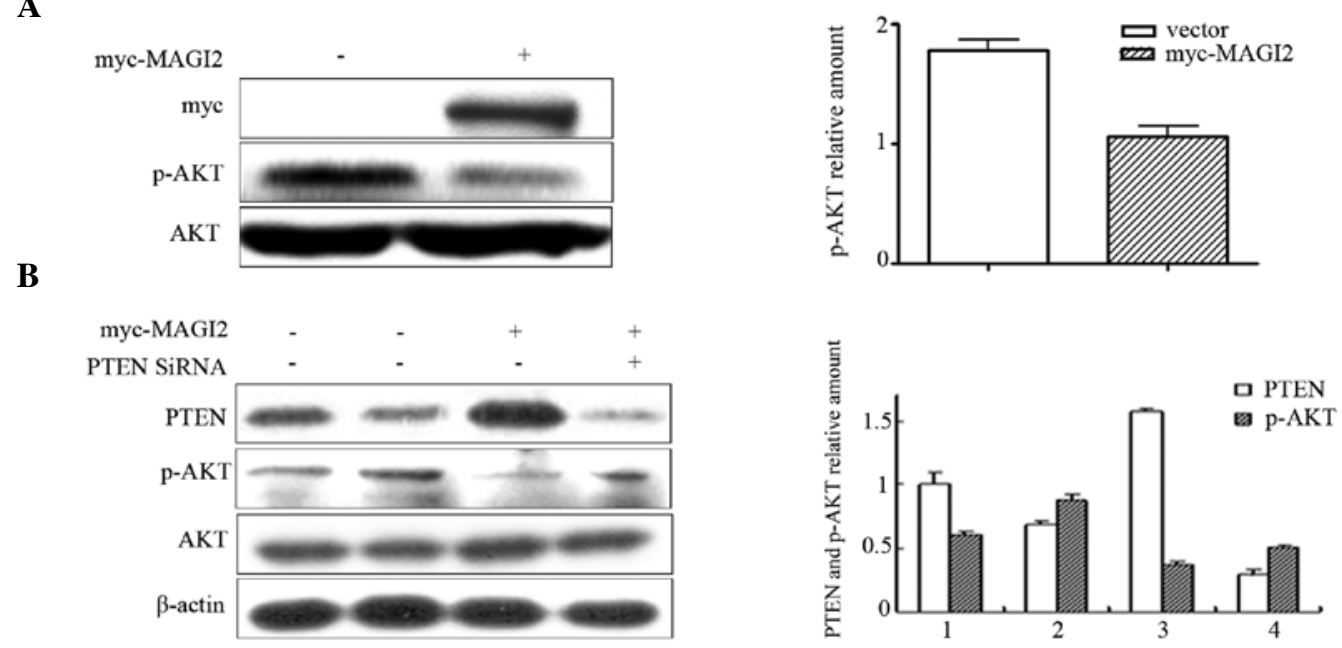

C

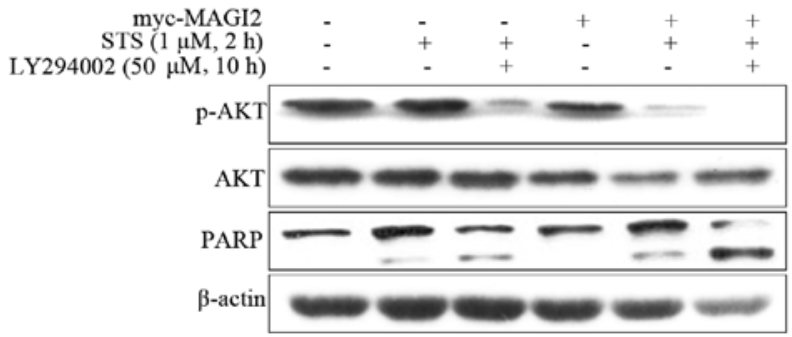

Figure 6. The activity of AKT was inhibited in response to membrane-associated guanylate kinase inverted 2 (MAGI2) overexpression. (A) The phosphorylation of AKT and total AKT protein level in the vector-7404 and myc-MAGI2-7404 cells. The chart on the right is the analysis of the quantitative representation of phosphorylated AKT (p-AKT) protein normalized to AKT. (B) Knockdown of PTEN by siRNA increased the phosphorylation level of AKT in the vector-7404 and myc-MAGI2-7404 cells. The chart on the right is the analysis of the quantitative ratios of p-AKT and PTEN relative to the amount of $\beta$-actin for the data mentioned above. Each bar represents the mean \pm SD of three independent experiments. (C) PARP and p-AKT were detected in the vector-7404 and myc-MAGI2 cells following treatment with $1 \mu \mathrm{M}$ STS for $2 \mathrm{~h}$ and after the addition of LY294002 for $10 \mathrm{~h}$. The result shown is from three independent experiments.

PTEN, we examined the intracellular distribution of MAGI2 and PTEN during STS-induced apoptosis under a confocal immunofluorescence microscope. The co-localization (orange) of MAGI2 and PTEN was observed in the cytoplasm of the myc-MAGI2-7404 cells, particularly beneath the plasma membrane when the myc-MAGI2-7404 cells were treated with $1 \mu \mathrm{M}$ STS for $2 \mathrm{~h}$. MAGI2 and PTEN were dispersed in the cytoplasm or nucleus of the vector-7404 cells (Fig. 5).

MAGI2 enhances the ability of PTEN to suppress the activation of $A K T$. AKT is a crucial downstream molecule of the PI3K pathway. Multiple factors regulate the PI3K/AKT signaling pathway, in which the negative feedback is modulated by PTEN. PTEN has phosphatase activity and dephosphorylates PIP3 to form PIP2, thus preventing the activation of AKT. To explore whether MAGI2 enhances the ability of PTEN to suppress the activation of AKT, we firstly observed the phosphorylation of AKT and total AKT protein levels in the vector-7404 and myc-MAGI2-7404 cells. The AKT phosphorylation level was lower in the myc-MAGI2-7404 cells than in the vector-7404 cells, despite the fact that no significant changes in total AKT levels were observed in these cell lines (Fig. 6A). MAGI2 overexpression caused PTEN protein levels to increase and phosphorylated AKT levels to decrease (Fig. 6B, lane 3). Furthermore, the reduction in PTEN expression in the presence of PTEN siRNA induced an increase in phosphorylated AKT levels in the myc-MAIG2-7404 cells, and there were no apparent change in total AKT protein levels in the vector-7404 and myc-MAGI2-7404 cells (Fig. 6B, lanes 2 and 4). Finally, to better explore whether AKT is involved in the MAGI2regulated apoptosis, the vector-7404 and myc-MAGI2-7404 cells were treated with LY294002 (a PI3K inhibitor), then STS-induced apoptosis was observed by detecting the cleavage of PARP. The results revealed that the phosphorylation level of AKT was decreased, and the cleavage of PARP was increased following incubation with $50 \mu \mathrm{M}$ LY294002 for $10 \mathrm{~h}$ and $1 \mu \mathrm{M}$ STS for $2 \mathrm{~h}$ in the myc-MAGI2-7404 cells compared with the vector-7404 cells (Fig. 6C, lanes 3 with 6). These results clearly indicate that the phosphorylation level of AKT negatively correlates with apoptosis. The activity of AKT was inhibited in response to MAGI2 overexpression, which played a role in the enhancement of STS-induced apoptosis by PTEN.

\section{Discussion}

Apoptosis is a highly regulated and coordinated cellular phenomenon involved in multiple key molecules $(23,24)$. In the present study, we found that MAGI2 overexpression increased PTEN levels; the change in PTEN protein levels occurred in the post-translational stage through enhancing its protein stability rather than elevating its mRNA level. Our results demonstrate that PTEN decreases the phosphorylation level of AKT; thus, MAGI2 may decrease the phosphorylation level of AKT by upregulating endogenous PTEN in its tumor suppressive action. 
The potential factors that influence the STS-induced apoptosis in tumors may lead to the development of more effective strategies and novel chemotherapeutic drugs for the treatment of patients suffering from cancer.

Increasing evidence indicates that higher levels of AKT activity are found in human glioblastoma cells compared with astrocytes and this may contribute to their resistance to apoptosis $(18,25)$. AKT overexpression is associated with poor prognosis, resistance to chemotherapy and the shortened survival time of cancer patients (26). PI3K activates AKT by phosphorylating PIP2 to form PIP3. In various tumor cells, the elevated activation of AKT has been attributed to mutations or the deletion of the PTEN tumor suppressor, which functions as a PIP3 lipid phosphatase and antagonizes PI3K signaling (16). Cells bearing PTEN mutation show an elevated level of phosphorylated AKT, increased proliferation and resistance to numerous apoptotic agents $(27,28)$. The PI3K signaling pathway has emerged as a critical route that regulates multiple cellular processes, including cell survival and proliferation in several cell types (29). AKT has been implicated in PI3K-mediated survival effects $(30,31)$. The transfection of fibroblasts and epithelial cells with constitutively active PI3K or AKT has been shown to result in the inhibition of apoptosis induced by c-Myc, UV radiation, TGF- $\beta$ and Fas activation (32-35).

It is known that PDZ domains are found in many types of proteins, including a family of membrane-associated scaffold proteins known as MAGUKs (36). MAGUK proteins contain multiple protein-protein interaction domains, including guanylate kinase, WW and PDZ domains, and they homodimerize and heterodimerize to form scaffolding complexes. The MAGUK family of scaffolding proteins, such as MAGI1, MAGI2, calcium/calmodulin-dependent serine protein kinase (CASK) and zona occludens-1 (ZO-1), link immunoglobin family receptors, including nephrin and cadherins in podocytes (37). PTEN has a C-terminal in which the last four amino acids encode a PDZ domain-binding motif (38). It has been reported that PTEN binds to MAGI2 through an interaction between the C-terminus of PTEN and the second PDZ domain of MAGI2 $(11,39)$. MAGI2, as a synaptic scaffolding molecule, has been reported to interact with $\beta$-catenin. A previous study demonstrated that vinculin controls PTEN protein levels by maintaining the interaction of the adherens junction protein, $\beta$-catenin, with the scaffolding protein, membrane-associated MAGI2 (15). The authors revealed a novel mechanism of PTEN regulation, whereby its protein level is controlled through the maintenance of $\beta$-catenin-MAGI 2 interaction by vinculin (15).

Our results demonstrated that at different concentrations of STS and different exposure times, STS induced apoptosis in the myc-MAGI2-7404 cells; the proportion of apoptotic cells was significant compared to the vector-7404 cells (Fig. 2). However, when PTEN siRNA was used, reducing the levels of PTEN, MAGI2 did not enhance the sensitivity of the human hepatocellular carcinoma cells to STS-induced apoptosis (Fig. 4). These results indicated that transfection with PTEN siRNA restored the effects of active AKT in myc-MAGI2-7404 cells.

To further explore the mechanism of PTEN upregulation, we examined PTEN protein stability; the half-life of PTEN was prolonged in the myc-MAGI2-7404 cells compared with the vector-7404 cells. These results indicated that MAGI 2 upregulated PTEN levels by enhancing its protein stability rather than elevating its mRNA level (Fig. 3). We also found that MAGI2 co-localized with PTEN beneath the plasma membrane and cytoplasm in response to STS in the myc-MAGI2-7404 cells (Fig. 5). This suggests that the MAGI-PTEN complex prevents the degradation PTEN and increases the PTEN protein level, resulting in the cells being more sensitive to STS. These results are consistent with those from our previous study, demonstrating the molecular association between PTEN and MAGI-2 (5).

Furthermore, phosphorylated AKT levels in the PTEN siRNA-transfected myc-MAIG2-7404 cells were higher compared to the cells not transfected with PTEN siRNA (Fig. 6B, compare lanes 4 and 3). These results suggested that PTEN acted as a critical molecule in the inhibition of the AKT signaling pathway. The PI3K inhibitor, LY294002, was then used to treat the vector-7404 and myc-MAGI2-7404 cells in the presence of STS. The results revealed that the phosphorylation of AKT was decreased, while the cleavage of PARP was increased in the myc-MAGI2-7404 cells compared with the vector-7404 cells (Fig. 6C, compare lanes 3 and 6). In the absence of MAGI2, STS induced apoptosis, but the apoptotic effect was not as pronounced as in the presence of MAGI2 (Fig. 6C, compare lanes 2 and 3 with lanes 5 and 6). The addition of LY294002 accelerated the STS-induced apoptosis, which suggested that the PTEN upregulation triggered by MAGI2 overexpression further influenced the phosphorylation levels of AKT, which elicited apoptosis or death signals. It can be hypothesized that MAGI2 upregulation enhances the sensitivity of cells harboring ectopic PTEN to STS. The function of cytoplasmic PTEN is the downregulation of AKT, which increases p27 levels and thereby leads to apoptosis (40). Our data demonstrated that transfection with the MAGI2 expression plasmid enhanced the sensitivity of human hepatocellular carcinoma cells to STS-induced apoptosis and identified cytoplasmic PTEN as an essential molecule in the MAGI2-induced apoptosis. Indeed, we observed a significant reduction in AKT phosphorylation following transfection with myc-MAGI2; these results suggest that AKT is involved in the MAGI2directed cell apoptosis as an effector downstream of PTEN.

In conclusion, the present study demonstrates that the overexpression of MAGI2 enhances the sensitivity of human hepatocellular carcinoma cells to STS-induced apoptosis. The MAGI2-PTEN complex may be a potential target in the development of novel chemotherapeutic drugs.

\section{Acknowledgements}

We are grateful for the gifts of the vector and myc-MAGI2 expression plasmid from Professor Yoshimi Takai. This study was supported by the National Natural Science Foundation of China (grant nos. 31070721 and 81000873).

\section{References}

1. Pawson T and Scott JD: Signaling through scaffold, anchoring, and adaptor proteins. Science 278: 2075-2080, 1997.

2. Burack WR and Shaw AS: Signal transduction: hanging on a scaffold. Curr Opin Cell Biol 12: 211-216, 2000.

3. Xu J, Paquet M, Lau AG, Wood JD, Ross CA and Hall RA: $\beta 1$-adrenergic receptor association with the synaptic scaffolding protein membrane associated guanylate kinase inverted-2 (MAGI2). Differential regulation of receptor internalization by MAGI2 and PSD-95. J Biol Chem 76: 41310-41317, 2001. 
4. Deng F, Price MG, Davis CF, Mori M and Burgess DL: Stargazin and other transmembrane AMPA receptor regulating proteins interact with synaptic scaffolding protein MAGI2 in brain. J Neurosci 26: 7875-7884, 2006.

5. Hu Y, Li Z, Guo L, et al: MAGI2 inhibits cell migration and proliferation via PTEN in human hepatocarcinoma cells. Arch Biochem Biophys 467: 1-9, 2007.

6. Hirao K, Hata Y, Ide N, et al: A novel multiple PDZ domaincontaining molecule interacting with $N$-methyl-D-aspartate receptors and neuronal cell adhesion proteins. J Biol Chem 273 21105-21110, 1998.

7. Wood JD, Yuan J, Margolis RL, et al: Atrophin-1, the DRPLA gene product, interacts with two families of WW domain-containing proteins. Mol Cell Neurosci 11: 149-160, 1998.

8. Nishimura W, Yao I, Iida J, Tanaka N and Hata Y: Interaction of synaptic scaffolding molecule and $\beta$-catenin. J Neurosci 22 : 757-765, 2002.

9. Ide N, Hata Y, Deguchi M, Hirao K, Yao I and Takai Y: Interaction of S-SCAM with neural plakophilin-related Armadillo-repeat protein/delta-catenin. Biochem Biophys Res Commun 256 456-461, 1999.

10. Yao I, Hata Y, Ide N, et al: MAGUIN, a novel neuronal membrane-associated guanylate kinase-interacting protein. J Biol Chem 274: 11889-11896, 1999.

11. Wu X, Hepner K, Castelino-Prabhu S, et al: Evidence for regulation of the PTEN tumor suppressor by a membrane-localized multi-PDZ domain containing scaffold protein MAGI2. Proc Natl Acad Sci USA 97: 4233-4238, 2000.

12. Subbaiah VK, Kranjec C, Thomasm and Banks L: PDZ domains: the building blocks regulating tumorigenesis. Biochem J 439 195-205, 2011

13. Morais Cabral JH, Petosa C, Sutcliffe MJ, et al: Crystal structure of a PDZ domain. Nature 382: 649-652,1996.

14. Valiente M, Andrés-Pons A, Gomar B, et al: Binding of PTEN to specific PDZ domains contributes to PTEN protein stability and phosphorylation by microtubule-associated serine/threonine kinases. J Biol Chem 280: 28936-28943, 2005.

15. Subauste MC, Nalbant P, Adamson ED and Hahn KM: Vinculin controls PTEN protein level by maintaining the interaction of the adherens junction protein beta-catenin with the scaffolding protein MAGI2. J Biol Chem 280: 5676-5681, 2005.

16. Maehama T and Dixon JE: The tumor suppressor, PTEN/ MMAC1, dephosphorylates the lipid second messenger, phosphatidylinositol 3,4,5-trisphosphate. J Biol Chem 273: 13375-13378, 1998.

17. Simpson L and Parsons R: PTEN: life as a tumor suppressor. Exp Cell Res 264: 29-41, 2001.

18. Haas-Kogan D, Shalev N, Wonget M, Mills G, Yount G and Stokoe D: Protein kinase B (PKB/AKT) activity is elevated in glioblastoma cells due to mutation of the tumor suppressor PTEN/MMAC. Curr Biol 8: 1195-1198, 1998.

19. Li DM and Sun H: PTEN/MMAC1/TEP1 suppresses the tumorigenicity and induces G1 cell cycle arrest in human glioblastoma cells. Proc Natl Acad Sci USA 95: 15406-15411, 1998.

20. Wang Y, Yang H, Liu H, Huang J and Song X: Effect of staurosporine on the mobility and invasiveness of lung adenocarcinoma A549 cells: an in vitro study. BMC Cancer 9: 174, 2009.

21. Gescher A: Analogs of staurosporine: potential anticancer drugs? Gen Pharmacol 31: 721-728, 1998.

22. Zhang Y, Yu S, Ou-Yang J, Xia D, Wang M and Li J: Effect of protein kinase $\mathrm{C}$ alpha, caspase-3, and survivin on apoptosis of oral cancer cells induced by staurosporine. Acta Pharmacol Sin 26: $1365-1372,2005$
23. Fesus L, Davies PJ and Piacentini M: Apoptosis: molecular mechanisms in programmed cell death. Eur J Cell Biol 56: 170-177, 1991.

24. Thornberry NA, Rano TA, Peterson EP, et al: A combinatorial approach defines specificities of members of the caspase family and granzyme B. Functional relationships established for key mediators of apoptosis. J Biol Chem 272: 17907-17911, 1997.

25. Sonoda Y, Ozawa T, Aldape KD, Deen DF, Berger MS and Pieper RO: AKT pathway activation converts anaplastic astrocytoma to glioblastoma multiforme in a human astrocyte model of glioma. Cancer Res 61: 6674-6678, 2001.

26. Ta HQ, Thomas KS, Schrecengost RS and Bouton AH: A novel association between p130Cas and resistance to the chemotherapeutic drug adriamycin in human breast cancer cells. Cancer Res 68: 8796-8804, 2008

27. Stambolic V, Suzuki A, de la Pompa JL, et al: Negative regulation of PKB/AKT-dependent cell survival by the tumor suppressor PTEN. Cell 95: 29-39, 1998.

28. Sun H, Lesche R, Li DM, et al: PTEN modulates cell cycle progression and cell survival by regulating phosphatidylinositol 3,4,5,-trisphosphate and AKT/protein kinase B signaling pathway. Proc Natl Acad Sci USA 96: 6199-6204, 1999.

29. Brunet A, Datta SR and Greenberg ME: Transcription-dependent and -independent control of neuronal survival by the PI3K-AKT signaling pathway. Curr Opin Neurobiol 11: 297-305, 2001

30. Dudek H, Datta SR, Franke TF, et al: Regulation of neuronal survival by the serine-threonine protein kinase AKT. Science 275: 661-665, 1997.

31. Franke TF, Kaplan DR and Cantley LC: PI3K: downstream AKTion blocks apoptosis. Cell 88: 435-437, 1997.

32. Kauffmann-Zeh A, Rodriguez-Viciana P, Ulrich E, Gilbert C, Coffer P, Downward J and Evan G: Suppression of c-Mycinduced apoptosis by Ras signalling through PI(3)K and PKB. Nature 385: 544-548, 1997

33. Kulik G, Klippel A and Weber MJ: Antiapoptotic signalling by the insulin-like growth factor I receptor, phosphatidylinositol 3-kinase, and AKT. Mol Cell Biol 17: 1595-1606, 1997.

34. Chen RH, Su YH, Chuang RL and Chang TY: Suppression of transforming growth factor-beta-induced apoptosis through a phosphatidylinositol 3-kinase/AKT-dependent pathway. Oncogene 17: 1959-1968, 1998.

35. Gibson S, Tu S, Oyer R, Anderson SM and Johnson GL: Epidermal growth factor protects epithelial cells against Fas-induced apoptosis. Requirement for AKT activation. J Biol Chem 274: 17612-17618, 1999.

36. González-Mariscal L, Betanzos A and Avila-Flores A: MAGUK proteins: structure and role in the tight junction. Semin Cell Dev Biol 11: 315-324, 2000.

37. Lehtonen S, Ryan JJ, Kudlicka K, Iino N, Zhou H and Farquhar MG: Cell junction-associated proteins IQGAP1, MAGI-2, CASK, spectrins, and $\beta$-actinin are components of the nephrin multiprotein complex. Proc Natl Acad Sci USA 102: 9814-9819, 2005.

38. Tsunoda S, Sierralta J, Sun Y, et al: A multivalent PDZ-domain protein assembles signalling complexes in a G-protein-coupled cascade. Nature 388: 243-249, 1997.

39. Tolkacheva T, Boddapati M, Sanfiz A, Tsuchida K, Kimmelman AC and Chan AM: Regulation of PTEN binding to MAGI 2 by two putative phosphorylation sites at threonine 382 and 383. Cancer Res 61: 4985-4989, 2001.

40. Planchon SM, Waite KA and Eng C: The nuclear affairs of PTEN. J Cell Sci 121: 249-253, 2007. 